\title{
Obstrucción duodenal y hemorragia digestiva alta como presentación inicial de una tuberculosis duodenal aislada
}

\author{
Edinson Meregildo-Rodríguez', Katherine Méndez-Florián² y Walter Espino-Saavedra ${ }^{1}$
}

\section{Duodenal obstruction and upper gastrointestinal bleeding as the initial presentation of an isolated duodenal tuberculosis}

Primary duodenal tuberculosis is rare, even in endemic regions. The diagnosis poses a major challenge and requires a high index of suspicion, supported by imaging studies, microbiology, and histopatology obtained by endoscopic or surgical biopsy. We report the case of a 31-year-old man, without HIV infection or any previous history of tuberculosis, who presented with duodenal obstruction. After exploratory laparotomy, he presented a duodenal stenosis and upper gastrointestinal bleeding. A total of four diagnostic procedures (one laparotomy and three endoscopies) were performed, all of which included biopsies. Only the last endoscopy made with the technique "biopsy upon biopsy" showed the presence of acid fast bacilli and granulomas. The diagnosis of tuberculosis was confirmed by polymerase chain reaction in duodenal tissue. There was no evidence of involvement of other organs by tuberculosis. The patient had an excellent therapeutic response.

Key words: Tuberculosis; duodenal obstruction; gastrointestinal hemorrhage.

Palabras clave: Tuberculosis; obstrucción duodenal; hemorragia gastrointestinal.

\section{Introducción}

\section{$\mathrm{L}$}

a tuberculosis (TBC) es un importante problema de salud pública en países en desarrollo ${ }^{1,2}$. En el mundo, se han incrementado los casos de TBC extra-pulmonar, en particular en los pacientes con infección por $\mathrm{VIH}^{1}$. Más de $50 \%$ de casos de TBC gastrointestinal ocurren en pacientes con VIH. Otros factores de riesgo para la TBC gastrointestinal son la leucemia y el uso crónico de antisecretores gástricos ${ }^{1,3,4}$.

La TBC gastrointestinal es infrecuente $(5 \%$ de todos los casos), y afecta más comúnmente a la región ileocecal ( $80-90 \%$ de casos) $)^{1,2,4,6}$. La TBC duodenal representa entre 0,5 y $2,5 \%$ de todos los casos de TBC gastrointestinal ${ }^{1-3,5-7}$. La TBC duodenal primaria y aislada (sin compromiso de otros órganos) es extremadamente infrecuente ${ }^{1-3,6,9}$. La mayoría de los casos de TBC duodenal ocurren en regiones endémicas, entre la tercera y cuarta década de la vida ${ }^{1,6,7}$.

Presentamos un caso de TB duodenal primaria que se presentó como una obstrucción intestinal y hemorragia digestiva alta.

\section{Caso clínico}

Varón de 31 años, nacido en Jaén (provincia ubicada en la selva baja de Perú), que residía hacía seis meses por motivo de trabajo, como albañil, en el distrito de San Martín de Porres (norte de Lima). Sin antecedentes médicos personales ni familiares de relevancia. Refirió vacunación con BCG durante niñez. Negó el consumo de leche o carne cruda, drogas o alcohol, pero manifestó conductas sexuales de riesgo, desde hacía dos años. Así mismo, refería haber perdido involuntariamente $10 \mathrm{~kg}$ durante los últimos cuatro meses. Inició un cuadro de 10 días de distensión y dolor abdominal, náuseas, eructos, y sensación de saciedad precoz. Posteriormente, se agregaron vómitos biliosos persistentes, y ausencia de deposiciones. El paciente negó haber presentado previamente sudoración nocturna, fiebre o tos. El día previo a su ingreso, se realizó una endoscopía que evidenció una estenosis duodenal. Al examen clínico de su ingreso se encontraba en regular estado general, con aspecto consuntivo (peso: $40 \mathrm{~kg}$, talla: 1,55 m, IMC: 16). PA: 110/70 mm Hg, FC: 124 lpm, FR: $18 \mathrm{rpm}$, temperatura axilar: $37,5^{\circ} \mathrm{C}$. El examen del sistema respiratorio y cardiovascular no presentaba nada relevante, salvo taquicardia. El abdomen se encontraba distendido, con ruidos hidroaéreos disminuidos, doloroso a la palpación de epigastrio y mesogastrio. Dentro de los exámenes auxiliares tenía un hemograma con leucocitos $14.800 / \mathrm{mm}^{3}$ (segmentados $74 \%$, eosinófilos $8 \%$, linfocitos $10 \%$ ), hemoglobina $12,9 \mathrm{~g} / \mathrm{dl}$, plaquetas $806.000 / \mathrm{mm}^{3}$. La proteína $\mathrm{C}$ reactiva en suero fue de $48 \mathrm{mg} / \mathrm{L}$ (valor normal $<6 \mathrm{mg} / \mathrm{L}$ ) y la velocidad de eritrosedimentación: 10 $\mathrm{mm} / \mathrm{h}$ (valor normal $<20 \mathrm{~mm} / \mathrm{h}$ ). La radiografía de tórax no mostraba alteraciones y la radiografía de abdomen simple (de pie y decúbito) mostró ausencia de niveles hidroaéreos. Se diagnósticó una obstrucción intestinal
${ }^{1}$ Hospital Regional Lambayeque, Perú.

${ }^{2}$ Universidad Nacional Pedro Ruiz Gallo, Lambayeque, Perú.

Los autores no tenemos conflicto de interés alguno que declarar. Sin financiamiento externo.

Recibido: 14 de junio de 2018 Aceptado: 23 de abril de 2019

Correspondencia a: Edinson Meregildo dante_meregildo@hotmail.com 


\section{Caso Clínico}

alta secundaria a una estenosis duodenal, por lo que se realizó una laparotomía exploratoria. En los hallazgos operatorios se encontró un segmento de yeyuno, a $30 \mathrm{~cm}$ del ángulo de Treitz, edematoso y con escasa fibrina y ganglios mesentéricos aumentados de tamaño que se extraen para estudio histológico. Al tercer día postoperatorio el paciente continuó sin deposiciones ni flatulencias, y con vómitos biliosos. Evolucionó con fiebre y contenido gástrico borráceo (negro). Se realizó una endoscopía digestiva alta que mostró edema de la mucosa de la segunda y tercera porción duodenal con múltiples úlceras de $<12$ $\mathrm{mm}$ no confluentes, lesiones nodulares con patrón en empedrado, y una estenosis de $50 \%$ de la luz duodenal (Figura 1). El estudio histopatológico de las biopsias duodenales y ganglionar no fueron concluyentes. Se realizó una TC abdominal que mostró una hemorragia gástrica y duodenal activa, con asas duodenales con leve dilatación y engrosamiento edematoso de sus paredes; y presencia de numerosos grupos ganglionares perigástricos, periduodenales y mesentéricos. El hígado, bazo, riñones y retroperitoneo mantenían sus características conservadas (Figura 2). A la tercera semana postoperatoria se realizó una nueva endoscopía con técnica "biopsia sobre biopsia" para una extracción más profunda de tejido duodenal para

Figura 1. Endoscopía a nivel de segunda y tercera porción duodenal. Mucosa edematosa con múltiples úlceras de $<12$ $\mathrm{mm}$ no confluentes, lesiones nodulares con patrón en empedrado y estenosis de la luz duodenal.

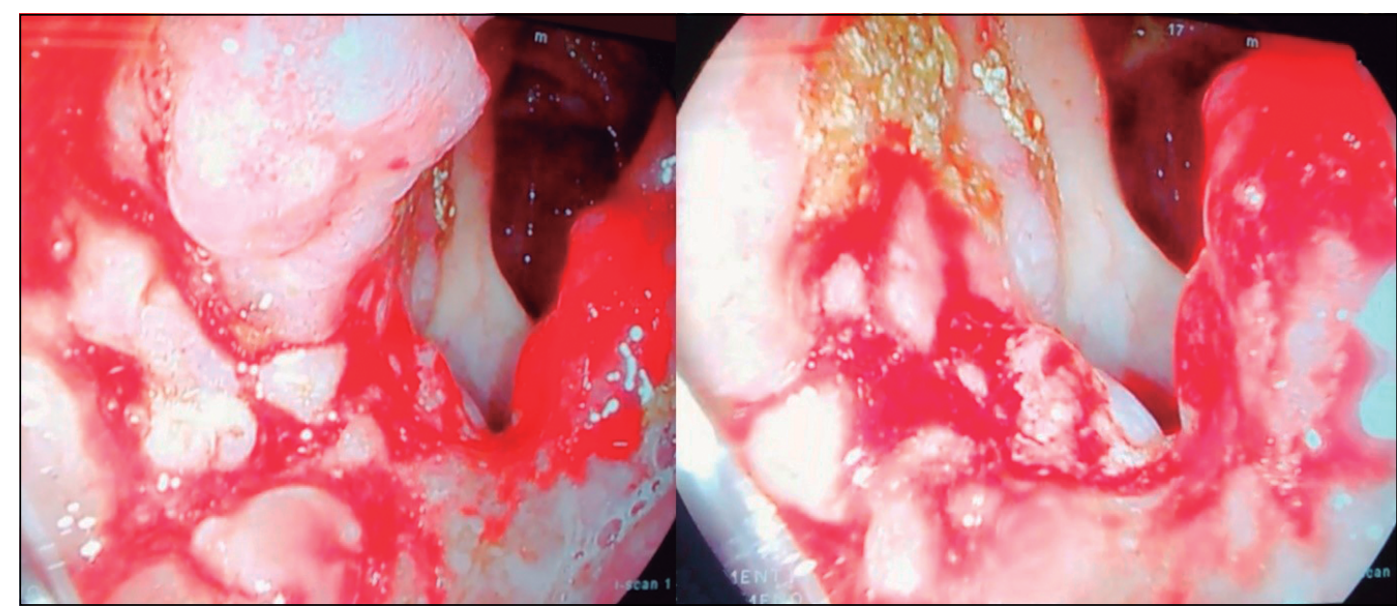

Figura 2. TC abdominal contrastada. Cortes axiales (A y $\mathbf{B})$ : estómago con sangrado activo (estrella amarilla) que se distribuye hasta segunda porción del duodeno (flechas rojas). Cortes coronales (C y D): unión de tercera y cuarta porción del duodeno (flechas azules) con estenosis y sangrado activo. Asas duodenales con dilatación y engrosamiento edematoso de sus paredes. Presencia de grupos ganglionares perigástricos, periduodenales y mesentéricos.

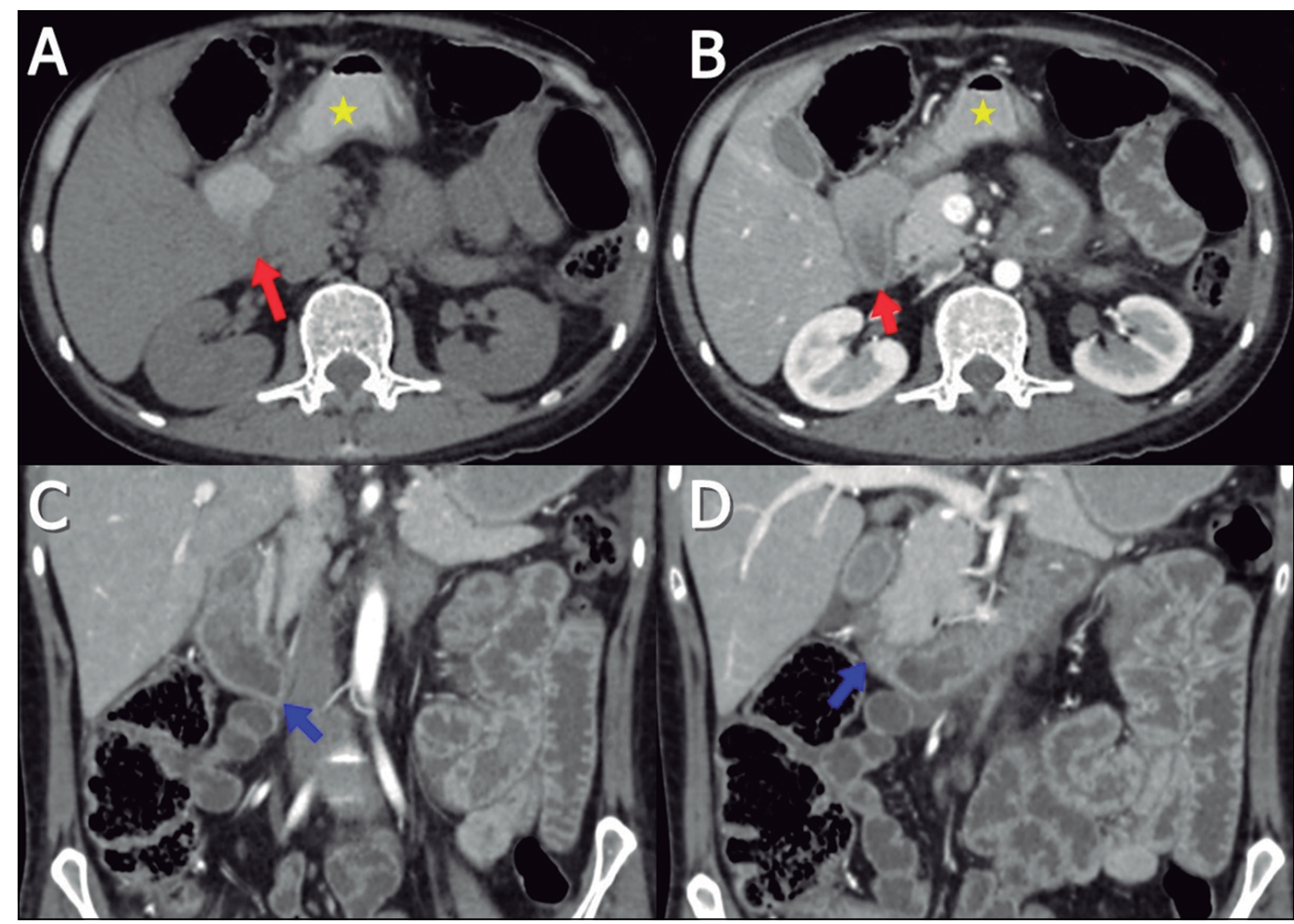


estudio. Posterior a este último procedimiento cursó con una hemorragia digestiva alta con repercusión hemodinámica y descenso de la hemoglobina de 8 a $4,7 \mathrm{~g} / \mathrm{dl}$. Se realizó manejo médico conservador con administración de volumen, inhibidores de bomba de protones, ácido tranexámico y lavado gástrico por SNG con solución salina a $35^{\circ} \mathrm{C}$. El estudio histológico de la última biopsia mostró una duodenitis crónica con presencia de células gigantes multinucleadas en la submucosa, y un granuloma no caseificante (Figura 3) y bacilos ácido alcohol resistentes (BAAR) (Figura 4), hallazgos compatibles con una TBC. Transcurrido un mes de la cirugía inició tratamiento antituberculoso estándar (isoniacida $5 \mathrm{mg} / \mathrm{kg} /$ día, rifampicina $10 \mathrm{mg} / \mathrm{kg} /$ día, pirazinamida $25 \mathrm{mg} / \mathrm{kg}$ / día y etambutol $20 \mathrm{mg} / \mathrm{kg} / \mathrm{día}$ ) y piridoxina $50 \mathrm{mg} / \mathrm{d}$, con buena evolución clínica.

La serología por método ELISA para VIH, HTLV-1, hepatitis B y C y VDRL/RPR fueron negativas. Las baciloscopias seriadas y el cultivo para $M$. tuberculosis en jugo gástrico, heces y orina fueron negativos. El coproparasitológico seriado fue positivo a Strongyloides stercoralis. No se realizó cultivo de muestras de tejido duodenal ni ganglionar. Tampoco se realizó PPD o test de tuberculina ya que no forma parte de la evaluación de rutina de un paciente con sospecha de TBC.

Posterior al alta, el paciente fue evaluado en dos ocasiones. La primera, dos semanas después del alta, donde se evidenció una notable mejoría del estado general, con una ganancia de $5 \mathrm{~kg}$ de peso. En esa oportunidad se recibió el resultado de la reacción de polimerasa en cadena positiva para $M$. tuberculosis, de una muestra tomada en la última endoscopía, procesada en el Instituto Nacional de Salud (INS) de Perú. Se indicó continuar con la terapia antituberculosa. La segunda evaluación, al final del segundo mes de tratamiento antituberculoso, el paciente ya presentaba recuperación clínica completa, con peso normal. Finalmente, nunca refirió algún contacto familiar o laboral con un caso de tuberculosis. No se realizó control endoscópico posterior al alta.

\section{Discusión}

La TBC duodenal es una de las formas más infrecuentes de compromiso gastrointestinal de la TBC, y representa una gran dificultad diagnóstica ${ }^{1-5}$. Su baja frecuencia se explica por varias razones: el efecto inhibitorio del ácido gástrico sobre M. tuberculosis, el tránsito intestinal rápido,

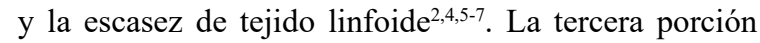
duodenal es la más frecuentemente afectada, seguida de la segunda porción ${ }^{1,7,12}$. Las lesiones duodenales pueden ser intrínsecas (60\% ulcerativa, 10\% hipertrófica, y 30\% úlcero-hipertrófica) o extrínsecas, secundaria a compresión por adenopatías $^{1,2,5,7}$. La mayoría de veces, la obstrucción

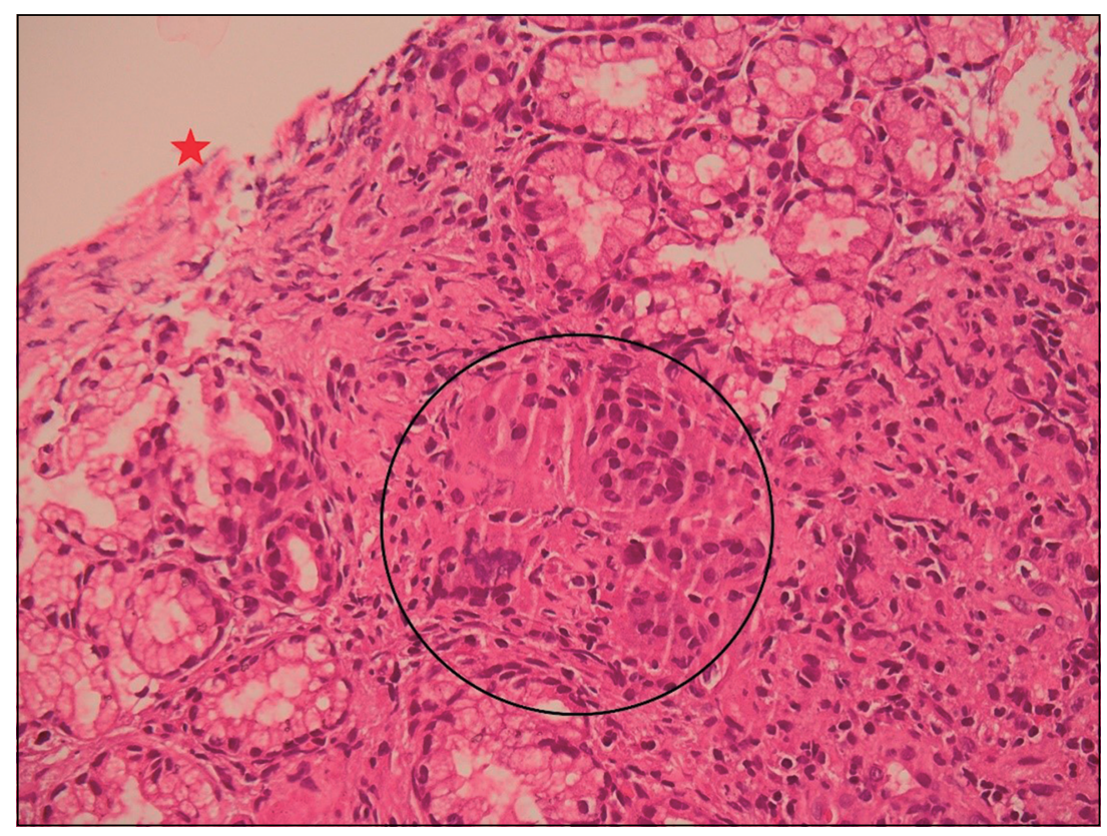

Figura 3. Microfotografía tomada con microscopio de luz (x1000). Duodenitis crónica severa (estrella roja); en la submucosa, presencia de células gigantes multinucleadas y un granuloma no caseificante. Muestra de biopsia duodenal con tinción de hematoxilina-eosina.

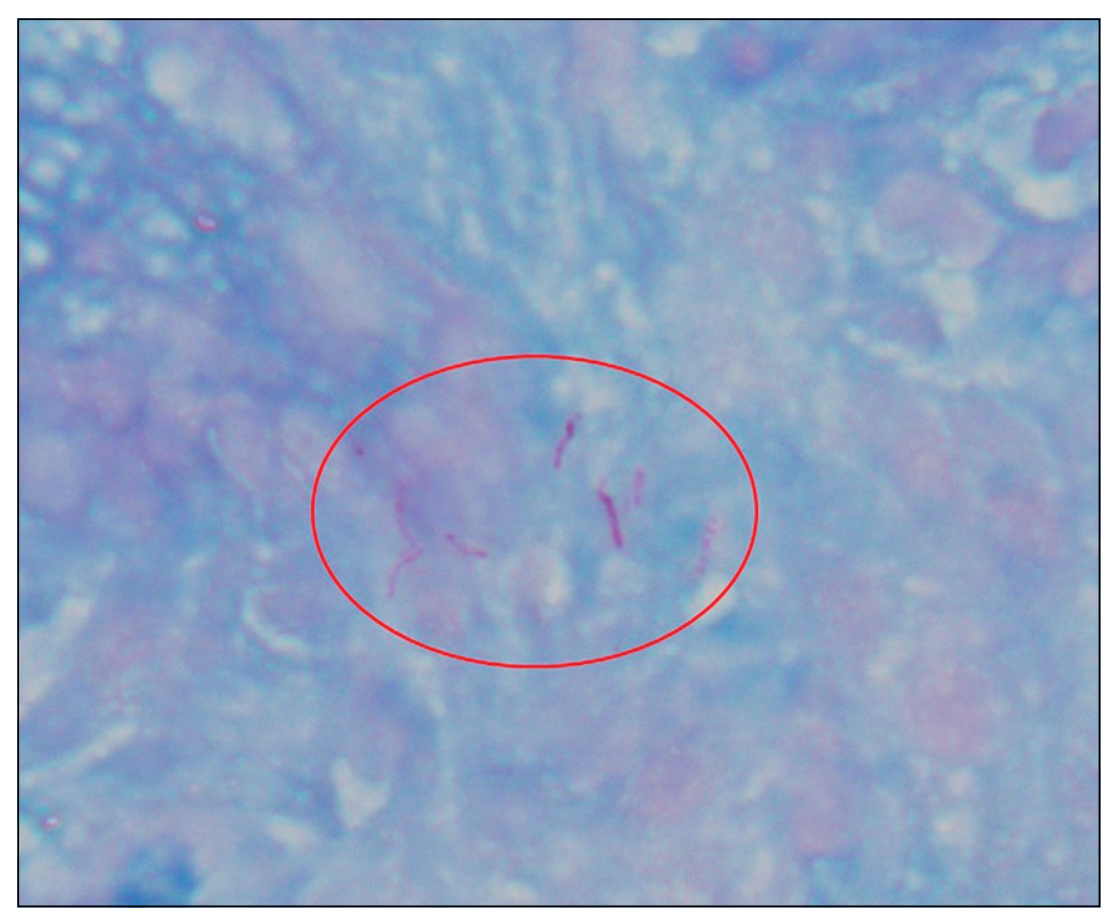

Figura 4. Microfotografía tomada con microscopio de luz (x1000). Presencia de bacilos ácido-alcohol resistentes. Muestra de biopsia duodenal con tinción de Ziehl Neelsen.

se debe tanto a procesos extrínsecos e intrínsecos ${ }^{6,7}$. En nuestro paciente, se demostró compromiso de la segunda, tercera y cuarta porciones duodenales, de tipo ulcerativo.

La TBC duodenal ocurre como resultado de una 
diseminación secundaria desde un foco pulmonar, en $10-50 \%$ de pacientes ${ }^{1,3-5}$; y generalmente, se presenta con compromiso masivo del tracto gastrointestinal ${ }^{5-7}$. Las posibles rutas de infección son una invasión directa a través de la mucosa, una diseminación hematógena o linfática, y por continuidad desde los ganglios linfáticos mesentéricos o celíacos ${ }^{2,4}$.

Clínicamente, la TBC duodenal produce síntomas de obstrucción del tracto gastrointestinal superior, que es la forma de presentación más común (60\% de casos), o de úlcera péptica (epigastralgia, o hemorragia digestiva) $)^{4,6-9}$. Los vómitos recurrentes son un signo cardinal $^{4,6}$. Son menos frecuentes la fiebre, ictericia, y complicaciones como perforación y fistulización ${ }^{1-6,8}$. La duración de los síntomas varía entre 2 días y 15 años ${ }^{4}$.

Debido a que las manifestaciones clínicas, radiológicas y endoscópicas de la TBC duodenal son inespecíficas, el diagnóstico requiere un alto índice de sospecha ${ }^{1-5,7}$. La biopsia endoscópica convencional de las úlceras tiene baja sensibilidad (0-10\%) ya que rara vez llega hasta la submucosa, localización predominante de los granulomas tuberculosos, y es inusual recuperar BAAR del material biopsiado $(4-6 \%)^{4-10}$. Para mejorar el rendimiento diagnóstico se recomienda tomar biopsias múltiples (8 a 10) y profundas usando técnicas especiales ("biopsia sobre biopsia" o "resección mucosa endoscópica") $)^{5,6-8}$. Es excepcional aislar M. tuberculosis en cultivo de lavado gástrico ${ }^{4}$. El diagnóstico definitivo se establece por histopatología (necrosis caseosa y células gigantes tipo Langhans) o microbiología (cultivo o tinción para BAAR) ${ }^{3-5}$. Los hallazgos patognomónicos (granulomas caseificantes o tinción BAAR positiva) se presentan en $<30 \%$ de $\operatorname{casos}^{5}$. La RPC en muestras clínicas tiene una sensibilidad de $72-90 \%$ y una especificidad de $100 \%{ }^{5}$. Sin embargo, algunas series reportan falsos negativos de hasta $40-65 \% \%^{4,6-8}$. La TBC duodenal es una enfermedad paucibacilar y la confirmación microbiológica no siempre es posible ${ }^{10}$.

En los casos que el diagnóstico es incierto, pese a haberse realizado los estudios pertinentes, en países endémicos se puede optar por el siguiente enfoque. Primero: si el índice de sospecha es alto, se justifica iniciar tratamiento antituberculoso empírico. La mejoría clínica ocurre en el lapso de dos semanas, y la curación de las úlceras y erosiones duodenales, en dos a tres meses. Segundo: si el índice de sospecha es bajo, se justifica la exploración quirúrgica (laparoscopia/laparotomía). De igual manera, si no ocurre mejoría con el tratamiento empírico en el tiempo previsto, se debe optar por la cirugía ${ }^{5,12}$.

El tratamiento de la TBC duodenal es médico y la respuesta es generalmente excelente ${ }^{1-3}$. Una vez que se ha establecido el diagnóstico, de ser posible, la cirugía se debe diferir hasta evaluar el resultado del tratamiento ${ }^{13}$. Sin embargo; el manejo dependerá de la presentación y las complicaciones asociadas. Si existe obstrucción, isquemia, perforación, hemorragia masiva, o peritonitis se optará por un tratamiento quirúrgico de emergencia ${ }^{5}$. Por el contrario, si la presentación es subaguda, se debe iniciar tratamiento antituberculoso tras confirmar el diagnóstico ${ }^{5}$. Por otra parte, en la mayoría de casos el diagnóstico de TBC duodenal se hace en el postoperatorio ${ }^{3}$. El uso de corticoesteroides para el tratamiento de una TBC gastrointestinal no tiene eficacia demostrada ${ }^{5}$.

El caso expuesto resulta de interés por varias razones. La presentación aguda del cuadro con una obstrucción intestinal que requirió una laparotomía exploratoria. Los síntomas obstructivos y la hemorragia gastroduodenal se asocian más comúnmente con malignidad y úlcera péptica. Sin embargo, como ocurrió con nuestro paciente, cuando no se encuentra malignidad, y no hay respuesta al tratamiento convencional para una úlcera péptica, se debe sospechar una TBC duodenal, entre otros diagnósticos diferenciales ${ }^{1,3}$. El diagnóstico diferencial debe incluir también enfermedad de Crohn y sarcoidosis ${ }^{8-11}$, enfermedades infrecuentes en nuestro medio.

Aunque la TBC duodenal primaria es extremadamente infrecuente, y pese a no haber compromiso pulmonar, ni antecedente de TBC o causa de inmunosupresión, en nuestro caso se mantuvo la sospecha de una TBC. A pesar que ya se habían realizado tres procedimientos invasores (dos endoscopías y una laparotomía), al persistir la sospecha clínica, se realizaron biopsias más profundas, que finalmente permitieron confirmar el diagnóstico.

\section{Resumen}

La tuberculosis duodenal primaria es muy infrecuente, incluso en regiones endémicas. El diagnóstico plantea un gran reto, y requiere un alto índice de sospecha, apoyado en estudios de imágenes, microbiología, e histopatología obtenida por biopsia endoscópica o quirúrgica. Presentamos el caso de un varón de 31 años, sin infección por VIH ni antecedente de tuberculosis, que debutó con una obstrucción duodenal. Posterior a una laparatomía exploradora presentó una estenosis duodenal y una hemorragia digestiva alta. Luego de varias biopsias no concluyentes, sólo la última, realizada con la técnica "biopsia sobre biopsia", demostró la presencia de granulomas con bacilos ácido-alcohol resistentes. El diagnóstico de tuberculosis fue confirmado por reacción de polimerasa en cadena de tejido duodenal. No se evidenció compromiso de otros órganos. La respuesta terapéutica fue excelente. 


\section{Referencias bibliográficas}

1.- Dahiya D, Garg M, Kaman L, Rana S, Rao C, Behera A. Duodenal tuberculosis - a rare case report and review of literature. Pol Przegl Chir 2013; 85: 464-6. doi: 10.2478/ pjs.2013.85.8.464.

2.- Chawla I, Aery V, Singh K, Singla B, Singh V. Duodenal tuberculosis presenting as gastric outlet obstruction. J Surg Case Rep 2012; 2012 (7):13. doi: 10.1093/ jscr/2012.7.13.

3.- Flores H B, Zano F, Ang E L, Estanislao N. Duodenal tuberculosis presenting as gastric outlet obstruction: a case report. World J Gastrointest Endosc 2011; 3: 16-9. doi:10.4253/ wjge.v3.i1.16.

4.- Rao Y G, Pande G K, Sahni P, Chattopadhyay
T K. Gastroduodenal tuberculosis management guidelines, based on a large experience and a review of the literature. Can J Surg 2004; 47: 364-8.

5.- Wong K, Marcon N, Calderwood S, Baron E. Tuberculous enteritis. In: UpToDate. Post TW (Ed), UpToDate, Waltham, MA. Disponible en: www.uptodate.com. Fecha de acceso: 15 de enero de 2018

6.- Puri A, Sachdeva S, Banka A, Sakhuja P. Tuberculosis of the duodenum: clinical presentation, diagnosis and outcome. Indian J Tuberc 2013; 60: 83-8.

7.- Chavhan G E, Ramakantan R. Duodenal tuberculosis: radiological features on barium studies and their clinical correlation in 28 cases J Postgrad Med 2003; 49: 214-7.

8.- De A, Lamoria S, Dhawan S, Agarwal S,
Sharma V. Duodenal tuberculosis: dig deep to diagnose. Trop Doct 2016; 46: 172-4. doi:10.1177/0049475515613921

9.- Gupta I, Parihar A, Dev G, Gillani Z. Duodenal tuberculosis. J K Science 2001; 3: 132-4.

10.- Gupta P, Guleria S, Mathur S R, Ahuja V. Gastroduodenal tuberculosis: a rare cause of gastric outlet obstruction. Surg J 2010; 5: 36-9.

11.- Nagi B, Lal A, Gupta P, Kochhar R, Sinha SK. Radiological findings in duodenal tuberculosis: a 15-year experience. Abdom Imaging 2015; 40: 1104-9. doi: 10.1007/s00261-014-0302-y.

12.- Robledo-Ogazón F, Mier y Díaz J, AlvaradoAparicio A, Vargas A. Estenosis duodenal secundaria a tuberculosis. Presentación de un caso y revisión de la literatura. Cir Ciruj 2003; 71: 475-8. 